\title{
Differences in Antipsychotic-Related Adverse Events in Adult, Pediatric, and Geriatric Populations
}

\author{
Hersh Sagreiya ${ }^{1}$, Yi-Ren Chen ${ }^{2}$, Narmadan A. Kumarasamy ${ }^{3}$, Karthik Ponnusamy ${ }^{4}$, Doris \\ Chen ${ }^{5}$, Amar K. Das 6 \\ 1. Radiology, University of Pittsburgh Medical Center 2. Department of Neurosurgery, Stanford \\ University Medical Center 3. Radiology, Montefiore Medical Center 4. Orthopedics, Western University 5. \\ Internal Medicine, Stanford University Medical Center 6. Healthcare and Life Sciences, IBM T.J. Watson \\ Research Center
}

$\square$ Corresponding author: Hersh Sagreiya, sagreiya@gmail.com

Disclosures can be found in Additional Information at the end of the article

\section{Abstract}

In recent years, antipsychotic medications have increasingly been used in pediatric and geriatric populations, despite the fact that many of these drugs were approved based on clinical trials in adult patients only. Preliminary studies have shown that the "off-label" use of these drugs in pediatric and geriatric populations may result in adverse events not found in adults. In this study, we utilized the large-scale U.S. Food and Drug Administration (FDA) Adverse Events Reporting System (AERS) database to look at differences in adverse events from antipsychotics among adult, pediatric, and geriatric populations. We performed a systematic analysis of the FDA AERS database using MySQL by standardizing the database using structured terminologies and ontologies. We compared adverse event profiles of atypical versus typical antipsychotic medications among adult (18-65), pediatric (age $<18)$, and geriatric $(>65)$ populations. We found statistically significant differences between the number of adverse events in the pediatric versus adult populations with aripiprazole, clozapine, fluphenazine, haloperidol, olanzapine, quetiapine, risperidone, and thiothixene, and between the geriatric versus adult populations with aripiprazole, chlorpromazine, clozapine, fluphenazine, haloperidol, paliperidone, promazine, risperidone, thiothixene, and ziprasidone $(\mathrm{p}<0.05$, with adjustment for multiple comparisons). Furthermore, the particular types of adverse events reported also varied significantly between each population for aripiprazole, clozapine, haloperidol, olanzapine, quetiapine, risperidone, and ziprasidone (Chi-square, $\mathrm{p}<10^{-6}$ ). Diabetes was the most commonly reported side effect in the adult population, compared to behavioral problems in the pediatric population and neurologic symptoms in the geriatric population. We also found discrepancies between the frequencies of reports in AERS and in the literature. Our analysis of the FDA AERS database shows that there are significant differences in both the numbers and types of adverse events among these age groups and between atypical and typical antipsychotics. It is important for clinicians to be mindful of these differences when prescribing antipsychotics, especially when prescribing medications off-label.

Categories: Psychiatry, Pediatrics, Epidemiology/Public Health

Keywords: antipsychotic drugs, adverse events, pediatrics, geriatrics, fda, drug adverse effects, typical antipsychotics, atypical antipsychotics, database, children

\section{Introduction}

While antipsychotic medications were initially approved based on clinical trials in adult 


\section{Cureus}

populations, they are commonly prescribed "off-label" in pediatric and geriatric populations [12]. In addition, they are increasingly being prescribed to children. Between the 1993 - 1998 and 2005 - 2009 time periods, visits including a prescription for antipsychotics per 100 people increased from 0.24 to 1.83 for children, 0.78 to 3.76 for adolescents, and 3.25 to 6.18 for adults; moreover, antipsychotics were included in $31.1 \%$ of youth visits to psychiatrists [3]. While antipsychotics are among the most effective drugs for the treatment of schizophrenia, mania, or acute psychotic reactions, these medications are often prescribed to children and adolescents for non-FDA approved indications, such as disruptive behaviors and aggression [45]. Similarly, antipsychotics are frequently used in the elderly and are prescribed to more than a quarter of Medicare patients in nursing homes, with common conditions including dementia, delirium, and behavioral disturbances [2]. However, the use of these medications may result in unanticipated adverse events that are specific to the pediatric and geriatric populations [6]. In our study, we sought to elucidate the differences in adverse events between pediatric, adult, and geriatric populations using the FDA's Adverse Events Reporting System (AERS), a database that has collected information about adverse events since 1998 [7]. AERS is the FDA's primary tool for post-marketing adverse event surveillance, with over 250,000 adverse event reports annually [8]. A key strength of the AERS database is the ability to analyze a massive dataset and discover potentially new information regarding drug-related adverse events warranting further investigation. For instance, a recent paper probed the AERS database and found a potential link between amisulpride, cyamemazine, and olanzapine and torsadogenic risk [9]. Drug manufacturers are required to submit adverse event reports, while healthcare providers can voluntarily submit information.

\section{Materials And Methods}

We initially imported AERS quarterly data from January 2004 to September 2008 into the MySQL program (v.1.2.17) (Oracle Corp., Redwood Shores, CA). A table was created that mapped all the various drug names for antipsychotics to a generic name and a drug class (typical vs. atypical) using RxNorm (U.S. National Library of Medicine, Bethesda, MD) and Micromedex ${ }^{\circledR}$ (Truven Health Analytics, Greenwood Village, CO) (Table 1). Next, we joined this table with the AERS drug table (matching by DRUGNAME), the AERS demo table (matching by ISR, which stands for individual safety report), and the AERS REAC table (also matching by ISR). We first retrieved the total number of adverse events associated with each drug name, generic name, and drug class. Next, we created a yearage variable (which standardized all ages in AERS to be reported in years using the AGE and AGE_COD variables) as well as the GNDR_COD variable (which was reported as either "M" or "F") in order to repeat this analysis on the following five subgroups: yearage $<18$ (pediatrics), $18 \geqslant$ yearage $\leqslant 65$ (adults), and yearage $>65$ (geriatrics), GNDR_COD = "M" (males), and GNDR_COD = "F" (females).

\begin{tabular}{lll}
\hline Drug Name & Generic Name & Drug Class \\
\hline Abilify & Aripiprazole & Atypicals \\
Aripiprazole & Aripiprazole & Atypicals \\
Chlorpromazine & Chlorpromazine & Typicals \\
Clozapine & Clozapine & Atypicals \\
Clozaril & Clozapine & Atypicals \\
Decazate & Fluphenazine & Typicals \\
Dozine & Chlorpromazine & Typicals
\end{tabular}




\section{Cureus}

\begin{tabular}{|c|c|c|}
\hline Fazalco & Clozapine & Atypicals \\
\hline Fentazin & Perphenazine & Typicals \\
\hline Fluphenazine & Fluphenazine & Typicals \\
\hline Fortunan & Haloperidol & Typicals \\
\hline Geodon & Ziprasidone & Atypicals \\
\hline Haldol & Haloperidol & Typicals \\
\hline Haloperidol & Haloperidol & Typicals \\
\hline Invega & Paliperidone & Atypicals \\
\hline Kentace & Haloperidol & Typicals \\
\hline Largactil & Chlorpromazine & Typicals \\
\hline Loxapac & Loxapine & Typicals \\
\hline Loxapine & Loxapine & Typicals \\
\hline Loxitane & Loxapine & Typicals \\
\hline Mellaril & Thioridazine & Typicals \\
\hline Mesoridazine & Mesoridazine & Typicals \\
\hline Moban & Molindone & Typicals \\
\hline Moditen & Fluphenazine & Typicals \\
\hline Navane & Thiothixene & Typicals \\
\hline Noxene & Thiothixene & Typicals \\
\hline Olanzapine & Olanzapine & Atypicals \\
\hline Orap & Pimozide & Typicals \\
\hline Ormazine & Chlorpromazine & Typicals \\
\hline Permitil & Fluphenazine & Typicals \\
\hline Perphenazine & Perphenazine & Typicals \\
\hline Pimozide & Pimozide & Typicals \\
\hline Primazine & Promazine & Typicals \\
\hline Prolixin & Fluphenazine & Typicals \\
\hline Promazine & Promazine & Typicals \\
\hline Quetiapine & Quetiapine & Atypicals \\
\hline Rideril & Thioridazine & Typicals \\
\hline Risperdal & Risperidone & Atypicals \\
\hline
\end{tabular}




\section{Cureus}

\begin{tabular}{lll} 
Risperidone & Risperidone & Atypicals \\
Serenace & Haloperidol & Typicals \\
Serentil & Mesoridazine & Typicals \\
Seroquel & Quetiapine & Atypicals \\
Sparine & Promazine & Typicals \\
Stelazine & Trifluoperazine & Typicals \\
Symbyax & Olanzapine & Atypicals \\
Thioridazine & Thioridazine & Typicals \\
Thiothixene & Thiothixene & Typicals \\
Thorazine & Chlorpromazine & Typicals \\
Trifluoperazine & Trifluoperazine & Typicals \\
\hline Trilafon & Promazine & Typicals \\
Vesprin & Triflupromazine & Typicals \\
Ziprasidone & Ziprasidone & Atypicals \\
Zyprexa & Olanzapine & Atypicals \\
\hline & & \\
\hline
\end{tabular}

\section{TABLE 1: List of Antipsychotic Medications Mapped to Generic Name and Drug Class}

Next, for each drug, we computed the percent of antipsychotic-related adverse events that the drug represented in each population. We then used the z-test of proportions to compare this percent for each drug in the following categories: pediatrics vs. adults, adults vs. geriatrics, and males vs. females. This process was conducted separately for typical and atypical drugs. This resulted in a $\mathrm{z}$-score and a $\mathrm{p}$-value for each comparison, which was then adjusted using a Bonferroni correction for multiple comparisons, making the significance threshold 0.05/26= $1.92 \times 10^{-3}$.

Afterward, we retrieved the count of each individual adverse event associated with each generic drug, ordered by the frequency of occurrence in each population. We made sure not to include irrelevant or vague side effects in our top results, excluding terms such as "DRUG INTERACTION,” “ACCIDENTAL EXPOSURE,” and “ACCIDENTAL DRUG INTAKE BY CHILD.” In order to compare the frequencies of the different adverse events in the adult, pediatric, and geriatric populations, we conducted a Chi-square test. For each drug, we selected the top five adverse events in adults and added a sixth column that contained the sum of all other adverse events. We chose the top five since this minimized the number of cells in the Chi-square calculation that contained an expected value less than 5 , which is not ideal for the Chi-square test. Next, we compared the frequency of these particular adverse events in the adult, pediatric, and geriatric populations using a 3 by 6 Chi-square table with 10 degrees of freedom, and we calculated p-values for each of seven major drugs-aripiprazole, clozapine, haloperidol, olanzapine, quetiapine, risperidone, and ziprasidone-using the R statistical program 


\section{Cureus}

(v2.12.2). We also used the MedRDA (Medical Directory for Regulatory Activities: International Federation of Pharmaceutical Manufacturers and Associations, Geneva, Switzerland) hierarchy to map MedDRA Preferred Terms (the default FDA coding) to high-level terms and determined the frequency of the high-level terms in the three populations.

Next, we took the list of the top five adverse events for the seven drugs in the three populations and used Medical Subject Headings (MeSH terms) to evaluate how many times a particular drug-adverse event combination was indexed in PubMed for the three populations. For instance, for the side-effect "TREMOR" for aripiprazole in the geriatric population, we would have used the following search term: "aripiprazole"[Substance Name] AND ("Aged"

[Mesh]) AND tremor. We then compared the number of reports in AERS and in the literature. For the drug, population, and adverse event combinations that had fewer than five reports in the literature, we manually examined the results to ensure their validity and highlighted the ones that we confirmed to have less than five reports.

\section{Results}

A summary of the populations we studied is shown in Table 2.

\begin{tabular}{lr} 
Category & Value \\
\hline Total number of patients & 61,380 \\
Mean age \pm SD & $45.7 \pm 20.0$ \\
Patients where age $<18$ & 3,578 \\
Patients where age $\geq 18$ and age $\leq 65$ & 32,660 \\
Patients where age $>65$ & 7,260 \\
Patients where age is not available & 17,882 \\
Male patients & 27,783 \\
Female patients & 29,780
\end{tabular}

\section{TABLE 2: Demographics: Summary of the Population}

The percentage of antipsychotic-related side effects was often significantly different in the pediatric, adult, and geriatric populations for atypical and typical antipsychotics as shown in Tables 3-4. 


\section{Cureus}

\begin{tabular}{|c|c|c|c|c|c|c|}
\hline \multirow{2}{*}{ Generic Name } & \multicolumn{3}{|c|}{$\%$ of Adverse Events } & \multicolumn{2}{|c|}{ p-value (vs. Adults) } & \multirow{2}{*}{ Statistical Significance } \\
\hline & Pediatrics & Adults & Geriatrics & Pediatrics & Geriatrics & \\
\hline Aripiprazole & 25.9 & 9.8 & 4.8 & 0 & 0 & Both \\
\hline Clozapine & 4.6 & 17.3 & 13.9 & 0 & $2.7 e^{-14}$ & Both \\
\hline Olanzapine & 16.1 & 26.0 & 25.6 & 0 & 0.23 & Pediatrics \\
\hline Paliperidone & 0.6 & 0.6 & 0.2 & 0.43 & $3.5 e^{-5}$ & Geriatrics \\
\hline Quetiapine & 24.4 & 27.2 & 26.1 & $2.6 e^{-5}$ & 0.019 & Pediatrics \\
\hline Risperidone & 23.3 & 14.4 & 27.5 & 0 & 0 & Both \\
\hline Ziprasidone & 5.1 & 4.6 & 1.9 & 0.077 & 0 & Geriatrics \\
\hline TOTALS & 100.0 & 100.0 & 100.0 & & & \\
\hline
\end{tabular}

\section{TABLE 3: Comparison of the Number of Adverse Events in Each Population for}

\section{Atypical Antipsychotics}

Results were statistically significant either for pediatrics vs. adults, geriatrics vs. adults, or both. The significance threshold was $0.05 / 26=1.92 \times 10^{-3}$. The $p$-values that $R$ found to be extremely low are labeled as " $0 . "$ Items that were statistically significant are in bold. 


\section{Cureus}

\begin{tabular}{|c|c|c|c|c|c|c|}
\hline \multirow{2}{*}{ Generic Name } & \multicolumn{3}{|c|}{$\%$ of Adverse Events } & \multicolumn{2}{|c|}{ p-value (vs. Adults) } & \multirow{2}{*}{ Statistical Significance } \\
\hline & Pediatrics & Adults & Geriatrics & Pediatrics & Geriatrics & \\
\hline Chlorpromazine & 20.3 & 17.1 & 12.1 & 0.066 & $3.31 e^{-6}$ & Geriatrics \\
\hline Fluphenazine & 0.3 & 5.1 & 2.4 & $4.3 e^{-5}$ & $4.39 \mathrm{e}^{-6}$ & Both \\
\hline Haloperidol & 64.9 & 56.3 & 72.8 & 0.0011 & 0 & Both \\
\hline Loxapine & 2.8 & 2.7 & 1.9 & 0.46 & 0.043 & -- \\
\hline Mesoridazine & 0.0 & 0.2 & 0.1 & 0.20 & 0.27 & -- \\
\hline Molindone & 0.9 & 0.3 & 0.6 & 0.017 & 0.018 & -- \\
\hline Perphenazine & 0.3 & 2.7 & 2.3 & 0.0038 & 0.19 & -- \\
\hline Pimozide & 3.4 & 1.6 & 1.3 & 0.0082 & 0.17 & -- \\
\hline Promazine & 0.6 & 2.8 & 0.1 & 0.0095 & $9.39 \mathrm{e}^{-10}$ & Geriatrics \\
\hline Thioridazine & 5.5 & 3.9 & 3.4 & 0.070 & 0.20 & -- \\
\hline Thiothixene & 0.0 & 4.5 & 0.9 & $4.9 e^{-5}$ & $1.24 \mathrm{e}^{-10}$ & Both \\
\hline Trifluoperazine & 0.9 & 2.9 & 2.0 & 0.017 & 0.031 & -- \\
\hline TOTALS & 100.0 & 100.0 & 100.0 & & & \\
\hline
\end{tabular}

\section{TABLE 4: Comparison of the Number of Adverse Events in Each Population for Typical Antipsychotics}

Results were statistically significant either for pediatrics vs. adults, geriatrics vs. adults, or both. The significance threshold was $0.05 / 26=1.92 \times 10^{-3}$. The $p$-values that $R$ found to be extremely low are labeled as " $0 . "$ Items that were statistically significant are in bold.

Eight antipsychotics were associated with a significant difference in the number of adverse events in the pediatric vs. adult populations, including aripiprazole, clozapine, fluphenazine, haloperidol, olanzapine, quetiapine, risperidone, and thiothixene. Ten antipsychotics were associated with a significant difference in the number of adverse events in the adult vs. geriatric populations, including aripiprazole, chlorpromazine, clozapine, fluphenazine, haloperidol, paliperidone, promazine, risperidone, thiothixene, and ziprasidone. When we compared the distributions of adverse events in the adult population to the pediatric and geriatric populations, Chi-square tests revealed that they were significantly different, as the pvalues were $4.33 \mathrm{e}^{-32}, 1.68 \mathrm{e}^{-92}, 2.60 \mathrm{e}^{-35}, 6.96 \mathrm{e}^{-106}, 4.50 \mathrm{e}^{-124}, 3.43 \mathrm{e}^{-65}$, and $1.35 \mathrm{e}^{-7}$, respectively, for aripiprazole, clozapine, haloperidol, olanzapine, quetiapine, risperidone, and ziprasidone. Tables comparing the number of reports in the literature to those in the AERS database for the top five adverse events in seven major antipsychotics revealed some outliers in the three populations, as evidenced by the reports with less than five cases in the literature

(Tables 5-6). 


\section{Cureus}

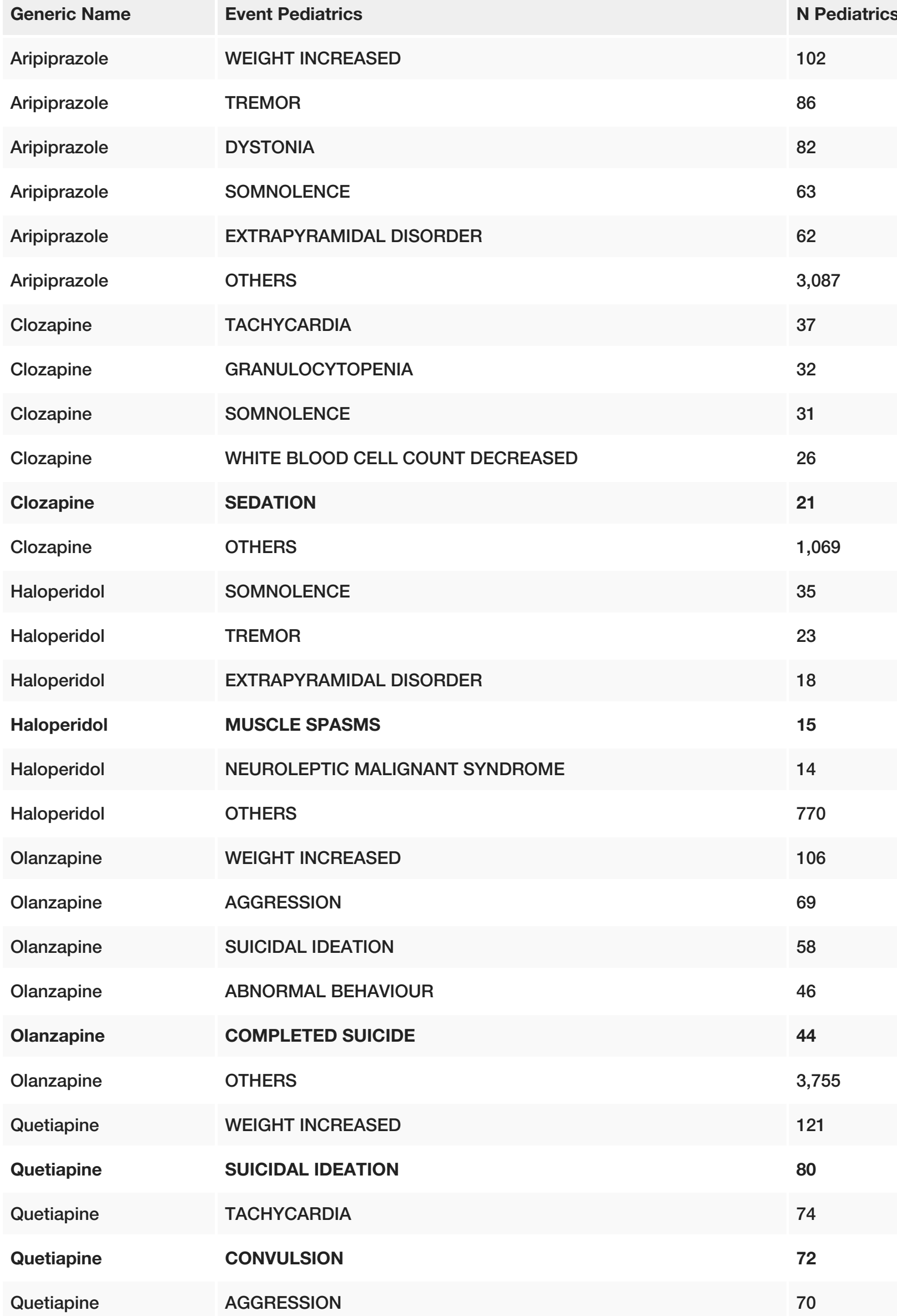




\section{Cureus}

$\begin{array}{lll}\text { Quetiapine } & \text { OTHERS } & 4,745 \\ \text { Risperidone } & \text { AGGRESSION } & 112 \\ \text { Risperidone } & \text { WEIGHT INCREASED } & 69 \\ \text { Risperidone } & \text { CONVULSION } & 66 \\ \text { Risperidone } & \text { SUICIDAL IDEATION } & 65 \\ \text { Risperidone } & \text { ABNORMAL BEHAVIOUR } & 54 \\ \text { Risperidone } & \text { OTHERS } & 4,015 \\ \text { Ziprasidone } & \text { DYSTONIA } & 26 \\ \text { Ziprasidone } & \text { SUICIDAL IDEATION } & 25 \\ \text { Ziprasidone } & \text { DEPRESSION } & 20 \\ \text { Ziprasidone } & \text { SUICIDE ATTEMPT } & 20 \\ \text { Ziprasidone } & \text { WEIGHT INCREASED } & 19 \\ \text { Ziprasidone } & \text { OTHERS } & 943\end{array}$

\section{TABLE 5: Top Adverse Events in the Pediatric Population}

The searches that have five or less PubMed articles are in bold.

\begin{tabular}{lll}
\hline Generic Name & Event Geriatrics & N Geriatrics \\
\hline Aripiprazole & TREMOR & 27 \\
Aripiprazole & NEUROLEPTIC MALIGNANT SYNDROME & 22 \\
Aripiprazole & PARKINSONISM & 21 \\
Aripiprazole & DEATH & 18 \\
Aripiprazole & GAIT DISTURBANCE & 15 \\
Aripiprazole & OTHERS & 1,087 \\
Clozapine & DEATH & 174 \\
Clozapine & PNEUMONIA & 100 \\
Clozapine & PYREXIA & 63 \\
Clozapine & SOMNOLENCE & 50 \\
Clozapine & FALL & 46 \\
Clozapine & OTHERS & 3,117
\end{tabular}




\section{Cureus}

\begin{tabular}{|c|c|c|}
\hline Haloperidol & AGITATION & 78 \\
\hline Haloperidol & CONFUSIONAL STATE & 75 \\
\hline Haloperidol & FALL & 68 \\
\hline Haloperidol & PYREXIA & 67 \\
\hline Haloperidol & DELIRIUM & 62 \\
\hline Haloperidol & OTHERS & 5,196 \\
\hline Olanzapine & FALL & 175 \\
\hline Olanzapine & CONFUSIONAL STATE & 142 \\
\hline Olanzapine & DIABETES MELLITUS & 138 \\
\hline Olanzapine & CEREBROVASCULAR ACCIDENT & 107 \\
\hline Olanzapine & PNEUMONIA & 100 \\
\hline Olanzapine & OTHERS & 9,494 \\
\hline Quetiapine & FALL & 155 \\
\hline Quetiapine & DEATH & 111 \\
\hline Quetiapine & CONFUSIONAL STATE & 107 \\
\hline Quetiapine & AGITATION & 103 \\
\hline Quetiapine & PNEUMONIA & 91 \\
\hline Quetiapine & OTHERS & 7,377 \\
\hline Risperidone & SOMNOLENCE & 161 \\
\hline Risperidone & DEATH & 159 \\
\hline Risperidone & CONFUSIONAL STATE & 152 \\
\hline Risperidone & FALL & 135 \\
\hline Risperidone & ASTHENIA & 117 \\
\hline Risperidone & OTHERS & 8,770 \\
\hline Ziprasidone & MYOCARDIAL INFARCTION & 15 \\
\hline Ziprasidone & COMA & 15 \\
\hline Ziprasidone & LOSS OF CONSCIOUSNESS & 11 \\
\hline Ziprasidone & SEDATION & 11 \\
\hline Ziprasidone & AGITATION & 11 \\
\hline Ziprasidone & OTHERS & 706 \\
\hline
\end{tabular}




\section{Cureus}

\section{TABLE 6: Top Adverse Events in the Geriatric Population}

The searches that have five or less PubMed articles are in bold.

Chi-square analysis was performed to compare the actual distribution of adverse events between the different populations for each drug. Seven commonly prescribed antipsychotics are presented in Table 7: aripiprazole, clozapine, haloperidol, olanzapine, quetiapine, risperidone, and ziprasidone.

\begin{tabular}{|c|c|c|c|c|c|}
\hline Generic Name & Event Adults & N Peds & N Adults & N Geriatrics & p-Value \\
\hline Aripiprazole & DIABETES MELLITUS & 11 & 288 & 10 & \\
\hline Aripiprazole & WEIGHT INCREASED & 102 & 235 & 13 & \\
\hline Aripiprazole & INSOMNIA & 11 & 227 & 12 & \\
\hline Aripiprazole & TREMOR & 86 & 177 & 27 & \\
\hline Aripiprazole & ANXIETY & 13 & 158 & 4 & \\
\hline Aripiprazole & OTHERS & 3,259 & 14,491 & 1,124 & $4.3 e^{-32}$ \\
\hline Clozapine & GRANULOCYTOPENIA & 32 & 611 & 27 & \\
\hline Clozapine & LEUKOPENIA & 11 & 390 & 20 & \\
\hline Clozapine & PYREXIA & 16 & 376 & 63 & \\
\hline Clozapine & DEATH & 0 & 342 & 174 & \\
\hline Clozapine & TACHYCARDIA & 37 & 309 & 17 & \\
\hline Clozapine & OTHERS & 1,120 & 29,669 & 3,249 & $1.7 \mathrm{e}^{-92}$ \\
\hline Haloperidol & DIABETES MELLITUS & 0 & 205 & 8 & \\
\hline Haloperidol & NEUROLEPTIC MALIGNANT SYNDROME & 14 & 196 & 44 & \\
\hline Haloperidol & SOMNOLENCE & 35 & 148 & 41 & \\
\hline Haloperidol & PYREXIA & 10 & 147 & 67 & \\
\hline Haloperidol & HYPERTENSION & 2 & 128 & 12 & \\
\hline Haloperidol & OTHERS & 814 & 14,528 & 5,374 & $2.6 \mathrm{e}^{-35}$ \\
\hline Olanzapine & DIABETES MELLITUS & 42 & 2,197 & 138 & \\
\hline Olanzapine & WEIGHT INCREASED & 106 & 1,464 & 64 & \\
\hline Olanzapine & HYPERTENSION & 21 & 906 & 56 & \\
\hline Olanzapine & ANCREATITIS & 27 & 865 & 3 & \\
\hline
\end{tabular}




\section{Cureus}

\begin{tabular}{|c|c|c|c|c|c|}
\hline Olanzapine & DIABETES MELLITUS NON-INSULIN-DEPENDENT & 6 & 720 & 21 & \\
\hline Olanzapine & OTHERS & 3,876 & 62,460 & 9,834 & $7.0 e^{-106}$ \\
\hline Quetiapine & DIABETES MELLITUS & 39 & 2,066 & 55 & \\
\hline Quetiapine & PANCREATITIS & 16 & 871 & 28 & \\
\hline Quetiapine & WEIGHT INCREASED & 121 & 662 & 18 & \\
\hline Quetiapine & SOMNOLENCE & 70 & 528 & 79 & \\
\hline Quetiapine & DIZZINESS & 41 & 473 & 75 & \\
\hline Quetiapine & OTHERS & 4,875 & 48,314 & 7,689 & $4.5 e^{-124}$ \\
\hline Risperidone & DIABETES MELLITUS & 20 & 614 & 34 & \\
\hline Risperidone & WEIGHT INCREASED & 69 & 390 & 15 & \\
\hline Risperidone & DEPRESSION & 46 & 327 & 41 & \\
\hline Risperidone & SOMNOLENCE & 47 & 292 & 161 & \\
\hline Risperidone & NEUROLEPTIC MALIGNANT SYNDROME & 19 & 262 & 37 & \\
\hline Risperidone & OTHERS & 4,180 & 32,300 & 9,206 & $3.4 e^{-65}$ \\
\hline Ziprasidone & DIABETES MELLITUS & 4 & 243 & 3 & \\
\hline Ziprasidone & WEIGHT INCREASED & 19 & 173 & 2 & \\
\hline Ziprasidone & ANXIETY & 15 & 116 & 5 & \\
\hline Ziprasidone & DEPRESSION & 20 & 105 & 3 & \\
\hline Ziprasidone & INSOMNIA & 12 & 104 & 6 & \\
\hline Ziprasidone & OTHERS & 983 & 10,316 & 750 & $1.3 e^{-7}$ \\
\hline
\end{tabular}

\section{TABLE 7: Chi-Square Analysis}

The distribution of antipsychotic-related adverse events was compared between the pediatric, adult, and geriatric populations for seven major antipsychotics. For each major antipsychotic drug, adverse events were ordered by their frequency in the adult population, the top five were selected (and the rest designated as "other"), and their distribution was compared using the Chisquare test. The resultant $p$-value is in the final column.

\begin{tabular}{|c|c|c|c|c|c|c|}
\hline Generic Name & Event Pediatrics & $\mathbf{N}$ & Event Adults & $\mathbf{N}$ & Event Geriatrics & $\mathbf{N}$ \\
\hline Chlorpromazine & $\begin{array}{l}\text { DRUG EXPOSURE } \\
\text { DURING PREGNANCY }\end{array}$ & 12 & DIABETES MELLITUS & 100 & WEIGHT DECREASED & 17 \\
\hline
\end{tabular}




\section{Cureus}

\begin{tabular}{|c|c|c|c|c|c|c|}
\hline Chlorpromazine & SOMNOLENCE & 9 & VOMITING & 59 & DIARRHOEA & 16 \\
\hline Chlorpromazine & AGGRESSION & 7 & $\begin{array}{l}\text { NEUROLEPTIC } \\
\text { MALIGNANT SYNDROME }\end{array}$ & 52 & DEHYDRATION & 15 \\
\hline Chlorpromazine & DRUG INEFFECTIVE & 6 & CONVULSION & 51 & PNEUMONIA & 14 \\
\hline Chlorpromazine & WEIGHT INCREASED & 6 & PYREXIA & 50 & SEPSIS & 13 \\
\hline Fluphenazine & ARRHYTHMIA & 1 & DIABETES MELLITUS & 26 & CONFUSIONAL STATE & 6 \\
\hline Fluphenazine & MYOCARDITIS & 1 & HYPERTENSION & 22 & $\begin{array}{l}\text { URINARY TRACT } \\
\text { INFECTION }\end{array}$ & 5 \\
\hline Fluphenazine & PYREXIA & 1 & WEIGHT INCREASED & 17 & SOMNOLENCE & 5 \\
\hline Fluphenazine & NA & 0 & HYPONATRAEMIA & 17 & SEDATION & 5 \\
\hline Fluphenazine & NA & 0 & HYPOTENSION & 16 & TACHYCARDIA & 3 \\
\hline Loxapine & $\begin{array}{l}\text { PROTHROMBIN LEVEL } \\
\text { DECREASED }\end{array}$ & 3 & PYREXIA & 15 & DYSPHAGIA & 5 \\
\hline Loxapine & $\begin{array}{l}\text { CONGENITAL } \\
\text { GENITOURINARY } \\
\text { ABNORMALITY }\end{array}$ & 2 & SEPSIS & 11 & CONFUSIONAL STATE & 4 \\
\hline Loxapine & CRYPTORCHISM & 2 & AGITATION & 10 & $\begin{array}{l}\text { DEPRESSED LEVEL } \\
\text { OF CONSCIOUSNESS }\end{array}$ & 4 \\
\hline Loxapine & $\begin{array}{l}\text { DRUG EXPOSURE } \\
\text { DURING PREGNANCY }\end{array}$ & 2 & LACTIC ACIDOSIS & 10 & SOMNOLENCE & 3 \\
\hline Loxapine & RENAL CYST & 2 & $\begin{array}{l}\text { BLOOD CREATINE } \\
\text { PHOSPHOKINASE } \\
\text { INCREASED }\end{array}$ & 9 & ANAEMIA & 3 \\
\hline Mesoridazine & NA & 0 & AGGRESSION & 4 & MALAISE & 1 \\
\hline Mesoridazine & NA & 0 & $\begin{array}{l}\text { EXCESSIVE } \\
\text { MASTURBATION }\end{array}$ & 3 & STOMATITIS & 1 \\
\hline Mesoridazine & NA & 0 & RASH PAPULAR & 3 & TARDIVE DYSKINESIA & 1 \\
\hline Mesoridazine & NA & 0 & SKIN ULCER & 3 & NA & 0 \\
\hline Mesoridazine & NA & 0 & RASH & 2 & NA & 0 \\
\hline Molindone & $\begin{array}{l}\text { NEUROLEPTIC } \\
\text { MALIGNANT SYNDROME }\end{array}$ & 2 & PRESCRIBED OVERDOSE & 3 & $\begin{array}{l}\text { HAEMOGLOBIN } \\
\text { DECREASED }\end{array}$ & 3 \\
\hline Molindone & MYOSITIS & 1 & CONVULSION & 3 & MYELOID LEUKAEMIA & 3 \\
\hline Molindone & PYREXIA & 1 & $\begin{array}{l}\text { DIABETES MELLITUS NON- } \\
\text { INSULIN-DEPENDENT }\end{array}$ & 2 & $\begin{array}{l}\text { PLATELET COUNT } \\
\text { DECREASED }\end{array}$ & 3 \\
\hline Molindone & VIRAL MYOSITIS & 1 & ANGER & 2 & $\begin{array}{l}\text { WHITE BLOOD CELL } \\
\text { COUNT INCREASED }\end{array}$ & 3 \\
\hline
\end{tabular}




\section{Cureus}

\begin{tabular}{|c|c|c|c|c|c|}
\hline Molindone & RASH & 1 & ABDOMINAL DISTENSION & 2 & TARDIVE DYSKINESIA \\
\hline Paliperidone & $\begin{array}{l}\text { NEUROLEPTIC } \\
\text { MALIGNANT SYNDROME }\end{array}$ & 10 & GALACTORRHOEA & 30 & DYSPNOEA \\
\hline Paliperidone & HEADACHE & 9 & $\begin{array}{l}\text { EXTRAPYRAMIDAL } \\
\text { DISORDER }\end{array}$ & 23 & $\begin{array}{l}\text { DEEP VEIN } \\
\text { THROMBOSIS }\end{array}$ \\
\hline Paliperidone & CONFUSIONAL STATE & 8 & AKATHISIA & 17 & TREMOR \\
\hline Paliperidone & PALPITATIONS & 8 & OEDEMA PERIPHERAL & 17 & RENAL FAILURE \\
\hline Paliperidone & DYSTONIA & 6 & DYSTONIA & 12 & CONFUSIONAL STATE \\
\hline Perphenazine & NA & 0 & VOMITING & 11 & DRUG INEFFECTIVE \\
\hline Perphenazine & NA & 0 & COMPLETED SUICIDE & 10 & HYPOTENSION \\
\hline Perphenazine & NA & 0 & DIABETES MELLITUS & 10 & INSOMNIA \\
\hline Perphenazine & NA & 0 & DRUG INTERACTION & 8 & $\begin{array}{l}\text { CEREBRAL } \\
\text { INFARCTION }\end{array}$ \\
\hline Perphenazine & NA & 0 & DRUG INEFFECTIVE & 8 & AGRANULOCYTOSIS \\
\hline Pimozide & WEIGHT INCREASED & 4 & CARDIAC ARREST & 12 & COMA \\
\hline Pimozide & DIARRHOEA & 4 & SUICIDE ATTEMPT & 8 & MEDICATION ERROR \\
\hline Pimozide & RECTAL HAEMORRHAGE & 4 & DRUG INTERACTION & 7 & $\begin{array}{l}\text { TOXIC SKIN } \\
\text { ERUPTION }\end{array}$ \\
\hline Pimozide & SOMNOLENCE & 3 & OVERDOSE & 6 & THROMBOCYTOPENIA \\
\hline Pimozide & ANOREXIA & 2 & ANXIETY & 6 & FALL \\
\hline Promazine & $\begin{array}{l}\text { NEONATAL DIABETES } \\
\text { MELLITUS }\end{array}$ & 1 & DIABETES MELLITUS & 33 & DRUG INTERACTION \\
\hline Promazine & PREMATURE BABY & 1 & PANCREATITIS & 15 & $\begin{array}{l}\text { METHYLMALONIC } \\
\text { ACIDURIA }\end{array}$ \\
\hline Promazine & DEATH & 1 & MYOCARDIAL INFARCTION & 15 & MUSCLE RIGIDITY \\
\hline Promazine & DIAPHRAGMATIC HERNIA & 1 & $\begin{array}{l}\text { BLOOD PRESSURE } \\
\text { DECREASED }\end{array}$ & 14 & CONFUSIONAL STATE \\
\hline Promazine & $\begin{array}{l}\text { PULMONARY } \\
\text { HYPOPLASIA }\end{array}$ & 1 & MYOCARDITIS & 14 & $\begin{array}{l}\text { PLATELET COUNT } \\
\text { INCREASED }\end{array}$ \\
\hline Thioridazine & NAUSEA & 8 & HEADACHE & 32 & CONFUSIONAL STATE \\
\hline Thioridazine & ANOREXIA & 8 & DIZZINESS & 29 & HYPERGLYCAEMIA \\
\hline Thioridazine & VOMITING & 5 & DEPRESSION & 25 & ANXIETY \\
\hline Thioridazine & ACHOLIA & 5 & ANXIETY & 24 & BACK PAIN \\
\hline Thioridazine & AGGRESSION & 5 & WEIGHT DECREASED & 22 & DEPRESSION \\
\hline
\end{tabular}




\section{Cureus}

\begin{tabular}{|c|c|c|c|c|c|}
\hline Thiothixene & NA & 0 & DIABETES MELLITUS & 63 & DYSPNOEA \\
\hline Thiothixene & NA & 0 & WEIGHT INCREASED & 56 & $\begin{array}{l}\text { CEREBROVASCULAR } \\
\text { ACCIDENT }\end{array}$ \\
\hline Thiothixene & NA & 0 & HEADACHE & 33 & NAUSEA \\
\hline Thiothixene & NA & 0 & PANCREATITIS & 33 & TARDIVE DYSKINESIA \\
\hline Thiothixene & NA & 0 & CHEST PAIN & 31 & $\begin{array}{l}\text { ANTICHOLINERGIC } \\
\text { SYNDROME }\end{array}$ \\
\hline Trifluoperazine & SEXUAL OFFENCE & 1 & DIABETES MELLITUS & 32 & $\begin{array}{l}\text { CEREBROVASCULAR } \\
\text { ACCIDENT }\end{array}$ \\
\hline Trifluoperazine & CONVULSION & 1 & INSOMNIA & 16 & CEREBRAL ATROPHY \\
\hline Trifluoperazine & DEPRESSION & 1 & DEPRESSION & 13 & $\begin{array}{l}\text { TRANSIENT } \\
\text { ISCHAEMIC ATTACK }\end{array}$ \\
\hline Trifluoperazine & INJURY & 1 & DIABETIC KETOACIDOSIS & 12 & TREMOR \\
\hline Trifluoperazine & MEDICATION ERROR & 1 & PANCREATITIS & 12 & $\begin{array}{l}\text { MYOCARDIAL } \\
\text { INFARCTION }\end{array}$ \\
\hline
\end{tabular}

TABLE 8: Number and Type of Events in Each Population for Minor Drugs

The top five adverse events for the seven major antipsychotics mapped to MedDRA high-level terms are listed in Table 9.

\begin{tabular}{|c|c|c|c|c|c|c|}
\hline Generic & Event Pediatrics & $\mathbf{N}$ & Event Adults & $\mathbf{N}$ & Event Geriatrics & $\mathbf{N}$ \\
\hline Aripiprazole & $\begin{array}{l}\text { Neurological signs and } \\
\text { symptoms NEC }\end{array}$ & 196 & $\begin{array}{l}\text { Neurological signs and } \\
\text { symptoms NEC }\end{array}$ & 577 & $\begin{array}{l}\text { Neurological signs and } \\
\text { symptoms NEC }\end{array}$ & 56 \\
\hline Aripiprazole & $\begin{array}{l}\text { Dyskinesias and movement } \\
\text { disorders NEC }\end{array}$ & 172 & $\begin{array}{l}\text { Dyskinesias and } \\
\text { movement disorders NEC }\end{array}$ & 445 & $\begin{array}{l}\text { General signs and } \\
\text { symptoms NEC }\end{array}$ & 36 \\
\hline Aripiprazole & $\begin{array}{l}\text { Disturbances in } \\
\text { consciousness NEC }\end{array}$ & 143 & Anxiety symptoms & 420 & Muscle tone abnormal & 33 \\
\hline Aripiprazole & $\begin{array}{l}\text { Physical examination } \\
\text { procedures }\end{array}$ & 133 & $\begin{array}{l}\text { General signs and } \\
\text { symptoms NEC }\end{array}$ & 408 & $\begin{array}{l}\text { Dyskinesias and } \\
\text { movement disorders } \\
\text { NEC }\end{array}$ & 33 \\
\hline Aripiprazole & $\begin{array}{l}\text { General signs and } \\
\text { symptoms NEC }\end{array}$ & 117 & $\begin{array}{l}\text { Physical examination } \\
\text { procedures }\end{array}$ & 402 & $\begin{array}{l}\text { Parkinson's disease } \\
\text { and parkinsonism }\end{array}$ & 29 \\
\hline Clozapine & $\begin{array}{l}\text { Disturbances in } \\
\text { consciousness NEC }\end{array}$ & 78 & White blood cell analyses & 1,222 & $\begin{array}{l}\text { General signs and } \\
\text { symptoms NEC }\end{array}$ & 193 \\
\hline Clozapine & White blood cell analyses & 56 & Neutropenias & 1,137 & $\begin{array}{l}\text { Death and sudden } \\
\text { death }\end{array}$ & 192 \\
\hline
\end{tabular}




\section{Cureus}

\begin{tabular}{|c|c|c|c|c|c|c|}
\hline Clozapine & Neutropenias & 52 & $\begin{array}{l}\text { Disturbances in } \\
\text { consciousness NEC }\end{array}$ & 952 & $\begin{array}{l}\text { Disturbances in } \\
\text { consciousness NEC }\end{array}$ & 145 \\
\hline Clozapine & $\begin{array}{l}\text { Rate and rhythm disorders } \\
\text { NEC }\end{array}$ & 42 & $\begin{array}{l}\text { General signs and } \\
\text { symptoms NEC }\end{array}$ & 887 & $\begin{array}{l}\text { Lower respiratory tract } \\
\text { and lung infections }\end{array}$ & 121 \\
\hline Clozapine & $\begin{array}{l}\text { Neurological signs and } \\
\text { symptoms NEC }\end{array}$ & 39 & $\begin{array}{l}\text { Neurological signs and } \\
\text { symptoms NEC }\end{array}$ & 831 & $\begin{array}{l}\text { Lower respiratory tract } \\
\text { infections NEC }\end{array}$ & 117 \\
\hline Haloperidol & $\begin{array}{l}\text { Disturbances in } \\
\text { consciousness NEC }\end{array}$ & 51 & $\begin{array}{l}\text { Disturbances in } \\
\text { consciousness NEC }\end{array}$ & 471 & $\begin{array}{l}\text { Neurological signs and } \\
\text { symptoms NEC }\end{array}$ & 246 \\
\hline Haloperidol & $\begin{array}{l}\text { Medication errors due to } \\
\text { accidental exposures }\end{array}$ & 50 & $\begin{array}{l}\text { Neurological signs and } \\
\text { symptoms NEC }\end{array}$ & 425 & $\begin{array}{l}\text { Disturbances in } \\
\text { consciousness NEC }\end{array}$ & 162 \\
\hline Haloperidol & Muscle tone abnormal & 39 & $\begin{array}{l}\text { General signs and } \\
\text { symptoms NEC }\end{array}$ & 381 & $\begin{array}{l}\text { General signs and } \\
\text { symptoms NEC }\end{array}$ & 141 \\
\hline Haloperidol & Dyssomnias & 35 & Breathing abnormalities & 278 & $\begin{array}{l}\text { Ventricular arrhythmias } \\
\text { and cardiac arrest }\end{array}$ & 140 \\
\hline Haloperidol & $\begin{array}{l}\text { Dyskinesias and movement } \\
\text { disorders NEC }\end{array}$ & 33 & Liver function analyses & 274 & Anxiety symptoms & 126 \\
\hline Olanzapine & $\begin{array}{l}\text { Suicidal and self-injurious } \\
\text { behavior }\end{array}$ & 198 & $\begin{array}{l}\text { Diabetes mellitus (incl } \\
\text { subtypes) }\end{array}$ & 2,403 & $\begin{array}{l}\text { Disturbances in } \\
\text { consciousness NEC }\end{array}$ & 374 \\
\hline Olanzapine & $\begin{array}{l}\text { Physical examination } \\
\text { procedures }\end{array}$ & 150 & $\begin{array}{l}\text { Physical examination } \\
\text { procedures }\end{array}$ & 2,016 & $\begin{array}{l}\text { Neurological signs and } \\
\text { symptoms NEC }\end{array}$ & 355 \\
\hline Olanzapine & $\begin{array}{l}\text { Neurological signs and } \\
\text { symptoms NEC }\end{array}$ & 134 & $\begin{array}{l}\text { General signs and } \\
\text { symptoms NEC }\end{array}$ & 1,556 & $\begin{array}{l}\text { General signs and } \\
\text { symptoms NEC }\end{array}$ & 303 \\
\hline Olanzapine & $\begin{array}{l}\text { Behavior and socialization } \\
\text { disturbances }\end{array}$ & 130 & $\begin{array}{l}\text { Disturbances in } \\
\text { consciousness NEC }\end{array}$ & 1,551 & $\begin{array}{l}\text { Non-site specific } \\
\text { injuries NEC }\end{array}$ & 201 \\
\hline Olanzapine & $\begin{array}{l}\text { General signs and } \\
\text { symptoms NEC }\end{array}$ & 126 & $\begin{array}{l}\text { Neurological signs and } \\
\text { symptoms NEC }\end{array}$ & 1,312 & Liver function analyses & 182 \\
\hline Quetiapine & $\begin{array}{l}\text { Suicidal and self-injurious } \\
\text { behavior }\end{array}$ & 235 & $\begin{array}{l}\text { Diabetes mellitus (incl } \\
\text { subtypes) }\end{array}$ & 2,432 & $\begin{array}{l}\text { Neurological signs and } \\
\text { symptoms NEC }\end{array}$ & 331 \\
\hline Quetiapine & $\begin{array}{l}\text { Neurological signs and } \\
\text { symptoms NEC }\end{array}$ & 205 & $\begin{array}{l}\text { General signs and } \\
\text { symptoms NEC }\end{array}$ & 1,531 & $\begin{array}{l}\text { Disturbances in } \\
\text { consciousness NEC }\end{array}$ & 292 \\
\hline Quetiapine & $\begin{array}{l}\text { Physical examination } \\
\text { procedures }\end{array}$ & 178 & $\begin{array}{l}\text { Neurological signs and } \\
\text { symptoms NEC }\end{array}$ & 1,514 & $\begin{array}{l}\text { General signs and } \\
\text { symptoms NEC }\end{array}$ & 221 \\
\hline Quetiapine & $\begin{array}{l}\text { General signs and } \\
\text { symptoms NEC }\end{array}$ & 172 & $\begin{array}{l}\text { Disturbances in } \\
\text { consciousness NEC }\end{array}$ & 1,386 & $\begin{array}{l}\text { Non-site specific } \\
\text { injuries NEC }\end{array}$ & 181 \\
\hline Quetiapine & $\begin{array}{l}\text { Disturbances in } \\
\text { consciousness NEC }\end{array}$ & 170 & $\begin{array}{l}\text { Suicidal and self-injurious } \\
\text { behavior }\end{array}$ & 1,120 & $\begin{array}{l}\text { Circulatory collapse } \\
\text { and shock }\end{array}$ & 170 \\
\hline Risperidone & $\begin{array}{l}\text { Behavior and socialization } \\
\text { disturbances }\end{array}$ & 217 & $\begin{array}{l}\text { General signs and } \\
\text { symptoms NEC }\end{array}$ & 966 & $\begin{array}{l}\text { Disturbances in } \\
\text { consciousness NEC }\end{array}$ & 41 \\
\hline
\end{tabular}




\section{Cureus}

\begin{tabular}{|c|c|c|c|c|c|c|}
\hline Risperidone & $\begin{array}{l}\text { Suicidal and self-injurious } \\
\text { behavior }\end{array}$ & 202 & $\begin{array}{l}\text { Disturbances in } \\
\text { consciousness NEC }\end{array}$ & 870 & $\begin{array}{l}\text { Neurological signs and } \\
\text { symptoms NEC }\end{array}$ & 351 \\
\hline Risperidone & $\begin{array}{l}\text { Neurological signs and } \\
\text { symptoms NEC }\end{array}$ & 194 & $\begin{array}{l}\text { Neurological signs and } \\
\text { symptoms NEC }\end{array}$ & 867 & Asthenic conditions & 245 \\
\hline Risperidone & $\begin{array}{l}\text { General signs and } \\
\text { symptoms NEC }\end{array}$ & 128 & $\begin{array}{l}\text { Diabetes mellitus (incl } \\
\text { subtypes) }\end{array}$ & 713 & $\begin{array}{l}\text { General signs and } \\
\text { symptoms NEC }\end{array}$ & 223 \\
\hline Risperidone & $\begin{array}{l}\text { Dyskinesias and movement } \\
\text { disorders NEC }\end{array}$ & 121 & $\begin{array}{l}\text { Physical examination } \\
\text { procedures }\end{array}$ & 622 & $\begin{array}{l}\text { Death and sudden } \\
\text { death }\end{array}$ & 190 \\
\hline Ziprasidone & $\begin{array}{l}\text { Suicidal and self-injurious } \\
\text { behavior }\end{array}$ & 61 & $\begin{array}{l}\text { Neurological signs and } \\
\text { symptoms NEC }\end{array}$ & 319 & $\begin{array}{l}\text { Disturbances in } \\
\text { consciousness NEC }\end{array}$ & 41 \\
\hline Ziprasidone & $\begin{array}{l}\text { General signs and } \\
\text { symptoms NEC }\end{array}$ & 53 & $\begin{array}{l}\text { General signs and } \\
\text { symptoms NEC }\end{array}$ & 300 & $\begin{array}{l}\text { Ventricular arrhythmias } \\
\text { and cardiac arrest }\end{array}$ & 41 \\
\hline Ziprasidone & $\begin{array}{l}\text { Behavior and socialization } \\
\text { disturbances }\end{array}$ & 52 & Anxiety symptoms & 283 & $\begin{array}{l}\text { Neurological signs and } \\
\text { symptoms NEC }\end{array}$ & 35 \\
\hline Ziprasidone & $\begin{array}{l}\text { Neurological signs and } \\
\text { symptoms NEC }\end{array}$ & 47 & $\begin{array}{l}\text { Diabetes mellitus (incl } \\
\text { subtypes) }\end{array}$ & 281 & $\begin{array}{l}\text { Dyskinesias and } \\
\text { movement disorders } \\
\text { NEC }\end{array}$ & 24 \\
\hline Ziprasidone & Anxiety symptoms & 41 & $\begin{array}{l}\text { Disturbances in } \\
\text { consciousness NEC }\end{array}$ & 280 & $\begin{array}{l}\text { Ischemic coronary } \\
\text { artery disorders }\end{array}$ & 23 \\
\hline
\end{tabular}

\section{TABLE 9: Number and Type of Events in Each Population for Major Drugs Organized by MedDRA® High-Level Terms}

NEC: not elsewhere classified

\section{Discussion}

Overall, it was evident that both the frequencies and types of adverse events found in the adult population do not fit the distribution found in the pediatric or geriatric populations. As has been seen in prior studies, diabetes mellitus was frequently the most commonly reported adverse event in adults [10], but this was not the case for either the pediatric or geriatric populations. One possible explanation for this is that since adults are more likely than children to have impaired fasting glucose in the first place (often due to a longer exposure to certain physiolologic factors, such as obesity and a sedentary lifestyle), they may be more predisposed to developing this complication. On the other hand, "weight increase" was frequently a topfive adverse effect for the major antipsychotic medications in children, consistent with prior meta-analyses [11]. Children were also more likely to exhibit side effects, such as "aggression," "abnormal behavior," and "suicidality," cognitive effects that may be seen more often in the developing brain. In particular, suicide attempts have previously been linked to antipsychotics in children with the AERS database [12]. For the geriatric population, neurological side effects, such as "confusional state" and "somnolence," figured more prominently. This suggests that the elderly, who are predisposed to neurological problems, may be more severely affected by the neurological sequelae of antipsychotics. In fact, the Clinical Antipsychotic Trials of Intervention Effectiveness-Alzheimer's Disease (CATIE-AD) trial, 
studying elderly patients with Alzheimer's disease, showed that atypical antipsychotics were associated with worsening cognitive function comparable to an additional year's worth of cognitive decline compared to placebo [13].

Although we also analyzed differences in high-level terms between pediatric, adult, and geriatric populations, we realized that going to the next higher level grouping for MedDRA terms was not particularly illustrative. For instance, how does one distinguish "Neurological signs and symptoms" from "Disturbances in consciousness," and what exactly constitutes "General signs and symptoms?" These were among the most commonly reported high-level terms.

Our analysis of the literature revealed that there were adverse events that frequently had reports in AERS; yet, these events were not commonly mentioned in the literature. In the adult population, amongst the top five adverse events for the seven major antipsychotics, only pancreatitis in patients taking quetiapine had fewer than five reports in the literature. The analyses for the pediatric and geriatric populations generated comparatively more adverse events that were not commonly found in the literature. The result for quetiapine in the geriatric population is interesting, given reports of its association with pneumonia [14].

The limitations of the FDA AERS database include the lack of information on the number of individuals taking the various antipsychotic medications in each age group, which could have served as a "denominator" in our study. Due to this lack of a denominator, when comparing the total number of adverse events across the pediatric, adult, and geriatric populations, it was difficult to determine whether variations in the relative distribution of adverse events between the three age groups was truly due to differences in the rate of adverse events rather than simply variations in prescription frequency. For instance, this could be related to prescription trends or when the medications were released. Fortunately, the issue of a denominator was not problematic when comparing the particular side effect profile between the three populations for any given drug. Another issue is the fact that the correlation of a particular medication with an adverse event does not necessarily prove causation. For instance, an individual who is prone to a particular adverse event may be more likely to take an antipsychotic. Another potential problem is recall bias, as a physician who knows a patient is taking a given drug may be more likely to report adverse events that are widely known to be associated with that drug. Nevertheless, the sheer volume of the AERS database and its vast scope make it a useful tool for studying drug-related adverse events.

\section{Conclusions}

Overall, we were able to show that there are significant differences in both the numbers and types of adverse events between the pediatric, adult, and geriatric populations. In addition, this study offers a number of drug and adverse event combinations for follow-up analysis. Given the fact that these medications were overwhelmingly tested on the adult population and are commonly prescribed off-label, it is imperative that clinicians remain mindful of these differences when prescribing these medications in populations for whom the drugs were never formally tested.

\section{Additional Information \\ Disclosures}

Human subjects: All authors have confirmed that this study did not involve human participants or tissue. Animal subjects: All authors have confirmed that this study did not involve animal subjects or tissue. Conflicts of interest: In compliance with the ICMJE uniform disclosure form, all authors declare the following: Payment/services info: All authors have 
declared that no financial support was received from any organization for the submitted work. Financial relationships: All authors have declared that they have no financial relationships at present or within the previous three years with any organizations that might have an interest in the submitted work. Other relationships: All authors have declared that there are no other relationships or activities that could appear to have influenced the submitted work.

\section{References}

1. Biederman J: Credibility crisis in pediatric psychiatry. Nature Neurosci. 2008, 11:1233. 10.1038/nn0908-983

2. Wang PS, Schneeweiss S, Avorn J, Fischer MA, Mogun H, Solomon DH, Brookhart MA: Risk of death in elderly users of conventional vs. atypical antipsychotic medications. N Eng J Med. 2005, 353:2335-41. 10.1056/NEJMoa052827

3. Olfson M, Blanco C, Liu SM, Wang S, Correll CU: National trends in the office-based treatment of children, adolescents, and adults with antipsychotics. Arch Gen Psychiatry. 2012, 69:1247-56. 10.1001/archgenpsychiatry.2012.647

4. Gareri P, Segura-García C, Manfredi VG, Bruni A, Ciambrone P, Cerminara G, De Sarro G, De Fazio P: Use of atypical antipsychotics in the elderly: a clinical review . Clin Interv Aging. 2014, 9:1363-73. 10.2147/CIA.S63942

5. Penfold RB, Stewart C, Hunkeler EM, Madden JM, Cummings JR, Owen-Smith AA, Rossom RC, Lu CY, Lynch FL, Waitzfelder BE, Coleman KJ, Ahmedani BK, Beck AL, Zeber JE, Simon GE: Use of antipsychotic medications in pediatric populations: what do the data say? . Curr Psychiatry Rep. 2013, 15:426. 10.1007/s11920-013-0426-8

6. Vitiello B, Correll C, van Zwieten-Boot B, Zuddas A, Parellada M, Arango C: Antipsychotics in children and adolescents: increasing use, evidence for efficacy and safety concerns. Eur Neuropsychopharmacol. 2009, 19:629-35. 10.1016/j.euroneuro.2009.04.008

7. Moore TJ, Cohen MR, Furberg CD: Serious adverse drug events reported to the Food and Drug Administration, 1998-2005. Arch Intern Med. 2007, 167:1752-59.

10.1001/archinte.167.16.1752

8. Gibbons RD, Segawa E, Karabatsos G, Amatya AK, Bhaumik DK, Brown CH, Kapur K, Marcus SM, Hur K, Mann JJ: Mixed-effects Poisson regression analysis of adverse event reports: the relationship between antidepressants and suicide. Stat Med. 2008, 27:1814-33. 10.1002/sim.3241

9. Poluzzi E, Raschi E, Koci A, Moretti U, Spina E, Behr ER, Sturkenboom M, De Ponti F: Antipsychotics and torsadogenic risk: signals emerging from the US FDA Adverse Event Reporting System database. Drug Saf. 2013, 36:467-79. 10.1007/s40264-013-0032-z

10. Kato Y, Umetsu R, Abe J, Ueda N, Nakayama Y, Kinosada Y, Nakamura M: Hyperglycemic adverse events following antipsychotic drug administration in spontaneous adverse event reports. J Pharm Health Care Sci. 2015, 1:15. 10.1186/s40780-015-0015-6

11. Seida JC, Schouten JR, Boylan K, Newton AS, Mousavi SS, Beaith A, Vandermeer B, Dryden DM, Carrey N: Antipsychotics for children and young adults: a comparative effectiveness review. Pediatrics. 2012, 129:e771-84. 10.1542/peds.2011-2158

12. Kimura G, Kadoyama K, Brown JB, Nakamura T, Miki I, Nisiguchi K, Sakaeda T, Okuno Y: Antipsychotics-associated serious adverse events in children: an analysis of the FAERS database. Int J Med Sci. 2015, 12:135-40. 10.7150/ijms.10453

13. Vigen CL, Mack WJ, Keefe RS, et al: Cognitive effects of atypical antipsychotic medications in patients with Alzheimer's disease: outcomes from CATIE-AD. Am J Psychiatry. 2011, 168:831839. 10.1176/appi.ajp.2011.08121844

14. Kuo CJ, Yang SY, Liao YT, Chen WJ, Lee WC, Shau WY, Chang YT, Tsai SY, Chen CC: Secondgeneration antipsychotic medications and risk of pneumonia in schizophrenia. Schizophr Bull. 2013, 39:648-57. 10.1093/schbul/sbr202 